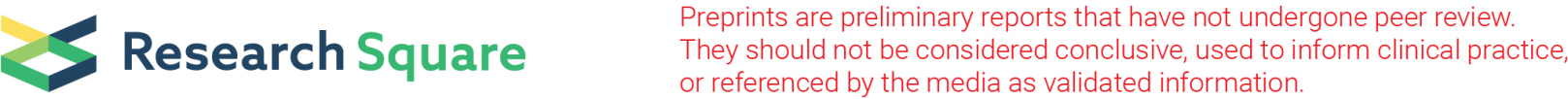

\section{Adipokine Human Resistin Promotes Obesity- associated Inflammatory Intervertebral Disc Degeneration via Pro-inflammatory Cytokine Cascade Activation}

Jae Hee Shin

Korea University

SeongHyun Park

University of Manchester

Hansang Cho

Sungkyunkwan University

Joo Han Kim

Korea University Medical Center

Hyuk Choi ( $\square$ hyuk76@korea.ac.kr)

Korea University

\section{Research Article}

Keywords: adipokine human resistin, intervertebral disc, inflammatory cytokines, extracellular matrix catabolic enzymes

Posted Date: June 25th, 2021

DOI: https://doi.org/10.21203/rs.3.rs-646684/v1

License: (c) (i) This work is licensed under a Creative Commons Attribution 4.0 International License.

Read Full License 


\section{Abstract}

Adipokine human Resistin (hResistin), is known to be associated with insulin resistance and secrete lowgrade pro-inflammatory cytokines in obesity. Although studies on low-grade inflammation of adipokine hResistin are known, studies on the effects and mechanisms of intervertebral disc degeneration (IVDD) are still lacking. Thus, we investigated the adipokine hResistin with or without pro-inflammatory cytokine IL-1 $\beta$ in intervertebral disc (IVD) cells such as human annulus fibrosus (hAF) and nucleus pulposus (hNP). The mean optical density changes in IL-1 $\beta$, IL-6, IL-8, MMP-1, MMP-3, and MMP-13, induced by the combined-hResistin and IL-1 $\beta$ stimulation on hAF cells, was significantly greater than that of the same induced by mono-IL-1 $\beta$ stimulation. Similarly, in the case of the mean optical density change of inflammatory mediators induced by the combined-hResistin and IL-1 $\beta$ stimulation on hNP cells was also significantly greater than that of the same induced by mono-IL-1 $\beta$ stimulation. These results improve understanding of hResistin on inflammatory IVDD but also with other obesity-related inflammatory diseases.

\section{Introduction}

In extension of the discovery of the low-grade pro-inflammatory cytokine activation nature of adipokines via the enhancement of secretion of pro-inflammatory mediators such as tumor necrosis factor (TNF)-a and interleukin (IL) families, which have been widely reported to be associated in the development of insulin resistance in obesity, understanding the mechanism of development and progression of the obesity-associated inflammatory intervertebral disc degeneration (IVDD) has received a strong attention to pave the way towards the development of better preventative and treatment measures for obesityrelated degenerative disc diseases ${ }^{1-3}$. Among various adipokines, leptin has been widely studied to investigate pathogenic mechanism of obesity-related inflammatory IVDD ${ }^{4-8}$. However, in contrast, pathogenic mechanism of obesity-related inflammatory IVDD-associated with other adipokines such as resistin has not been widely investigated. In 2016 Liu et al. reported the upregulation of expression of a disintegrin and metalloproteinase with thrombospondin motifs (ADAMTS) -5 via activation of p38 MAPK pathway induced by exposure of Resistin on rat nucleus pulposus (NP) cells ${ }^{9}$. ADAMTS-5 is widely reported to contribute IVDD ${ }^{10}$. In the following year, 2017, Li et al. reported a significant increase of expression of one of the macrophage inflammatory protein, CCL4, via activation of p38 mitogenactivated protein kinase (MAPK) and nuclear factor kappa B (NF-KB) signaling pathways induced by exposure of hResistin on hNP cells ${ }^{11}$. However, to the best of our knowledge, the correlation between the low-grade inflammation inducing nature of hResistin and the progression of inflammatory IVDD, which is associated with the promotion of various pro-inflammatory cytokine cascade activation, has not been previously reported. Hence, in this study, the promoting effect of hResistin on the promotion of progression of inflammatory disc degeneration-associated IL-1 $\beta$ pro-inflammatory cytokine cascade activation has been investigated.

\section{Materials And Methods}




\section{Isolation and Culture of hIVD Cells}

Human IVD cells were isolated from disc tissues of six patients (four males and two females) with degenerative spinal disease (Pfirrmann degenerative grades II-III) during elective surgery. Disc tissue collection was conducted with the approval of the institutional review board (KUGH170208-001) of Korea University Hospital, and informed consent was obtained from the subjects. All methods were performed in accordance with the guidelines and regulation of the Korea University Hospital Human Ethics Committee. Tissue specimens were placed in Ham's F-12 medium (Gibco-BRL, Grand Island, NY), containing $5 \%$ fetal bovine serum (FBS; Gibco-BRL) and $1 \%$ penicillin/streptomycin (P/S; Gibco-BRL), and then washed with phosphate-buffered saline (PBS; Gibco-BRL). Both annulus fibrosus (AF) and NP sections were minced and digested for $1 \mathrm{~h}$ at $37^{\circ} \mathrm{C}$ in an incubator under gentle stirring in F-12 medium containing $5 \% \mathrm{FBS}, 1 \% \mathrm{P} / \mathrm{S}$, and $0.2 \%$ pronase (Calbiochem, La Jolla, CA, USA). Subsequently, the minced AF and NP tissues were incubated overnight with $0.025 \%$ collagenase I (Roche Diagnostics, Mannheim, Germany). After overnight incubation, the cells were filtered through a sterile nylon mesh (70$\mu \mathrm{m}$ pore size) and centrifuged $(676 \times g, 5 \mathrm{~min})$. Both AF and NP pellets were resuspended in F-12 medium supplemented with $10 \% \mathrm{FBS}$ and $1 \% \mathrm{P} / \mathrm{S}$, and then cultured at $37^{\circ} \mathrm{C}$ in a humidified atmosphere containing $5 \% \mathrm{CO}_{2}$.

\section{Cell viability assay (WST-1 assay)}

Followed by the growth of IVD cells preparing to be confluent, the subsequent overnight serum starvation using $1 \mathbb{Q} / \mathrm{mL}$ of mitomycin $\mathrm{C}$ reagent was applied to inhibit progression of cell cycle. The cell viability of hIVD was measured by WST-1 colorimetric assay (ez-1000; Daeillab) followed by stimulation of hResistin (Peprotech) at five-different concentrations such as 25, 50, 100, 200, and $400 \mathrm{ng} / \mathrm{mL}$ for $48 \mathrm{~h}$.

Subsequently $10 \mu \mathrm{L}$ of WST-1 reagent was added into the resultant hResistin stimulated samples, which were seeded into a 96-well plate at a concentration of $3 \times 10^{3} /$ well in $200 \mu \mathrm{L}$ culture medium, followed by incubation for $4 \mathrm{~h}$ at $37^{\circ} \mathrm{C}$. Lastly, the absorbance values of the incubated samples were recorded at 450 nm using the spectrophotometer.

\section{Cell cytotoxicity assay (LDH and Live/Dead assay)}

To evaluate the possible cytotoxicity effects, cell cytotoxicity was verified with respect to different concentrations $(0,25,50,100,200$, and $400 \mathrm{ng} / \mathrm{mL})$ of hResistin using lactate dehydrogenase (LDH; Roche) and Live/Dead assay (Calcein-AM and Ethidium Homodimer-1; Invitrogen). $5 \times 10^{4}$ of both hAF and hNP cells were seeded in a 35-mm culture dish (SPL) with subsequent mono-hResistin stimulation. The culture supernatant (conditioned medium; CM) was collected after $48 \mathrm{~h}$ for LDH assay and the adhesive cells were subjected to Calcein-AM and Ethidium Homodimer-1 stimulation in accordance with the manufacturer's procedure.

\section{Stimulation of reagents on hIVD cells}

The cultured hAF and hNP cells were subjected to stimulation of combined-hResistin $(100 \mathrm{ng} / \mathrm{ml})$ and IL$1 \beta(1 \mathrm{ng} / \mathrm{ml})$ or mono-IL-1 $\beta(1 \mathrm{ng} / \mathrm{ml})$ for $48 \mathrm{~h}$. Prior to the stimulation process, the both $\mathrm{hAF}$ and $\mathrm{hNP}$ 
cells, seeded in six-well plates with stabilization for $24 \mathrm{~h}$, were undergone serum starvation for $24 \mathrm{~h}$. The schematic design and timeline of experiment for stimulation process is shown in Fig. 1 below.

\section{Real-time qRT-PCR}

Total RNA was extracted using TRIzol reagent (Invitrogen, Carlsbad, CA, USA). RNA (2,000 ng) was used to synthesize cDNA using a reverse transcription kit (Toyobo FSQ 101; Toyobo Co., Ltd., Osaka, Japan), in accordance with the manufacturer's protocol. Real-time PCR amplification was performed as follows: 40 cycles at $95^{\circ} \mathrm{C}$ for $15 \mathrm{~s}$ for denaturation and $60^{\circ} \mathrm{C}$ for $60 \mathrm{~s}$ for annealing and elongation. The primer sequences used were as follows: pro-IL-1 $\beta$, forward: 5'-TTCGACACATGGGATAACGAGG-3'; reverse: 5'TTTTTGCTGTGAGTCCCGGAG-3'; Caspase-1, forward: 5'-TTTCCGCAAGGTTCGATTTTCA-3'; reverse: 5'GGCATCTGCGCTCTACCATC-3'; NLRP3: forward: 5'-CCACAAGATCGTGAGAAAACCC-3'; reverse: 5'CGGTCCTATGTGCTCGTCA-3'; GAPDH: forward: 5'-CTGGGCTACACTGAGCACC-3'; reverse: 5'AAGTGGTCGTTGAGGGCAATG-3'. Gene expression was normalized to that of GAPDH.

\section{Western Blot Analysis}

Total protein was extracted and evaluated using a bicinchoninic acid assay. A sodium dodecyl sulfate (SDS) poly-acrylamide gel (10\%) was loaded with $40 \mu \mathrm{g}$ of total protein, followed by electrophoresis (SDS-PAGE). The resolved proteins were then transferred to polyvinylidene fluoride (PVDF) membranes (Bio-Rad), which were subsequently blocked with $5 \%$ skim milk or $3 \%$ BSA and incubated overnight at $4{ }^{\circ} \mathrm{C}$ with $5 \%$ BSA in TBST with the primary antibody. Immunolabeling was detected using ECL reagent (Invitrogen). The antibodies used for analysis were anti-phospho-p65, anti-p65, and anti- $\beta$-actin antibodies, which were purchased from Santa Cruz Biotechnology (1:1,000; Dallas, TX, USA), and antiphospho-p38 and anti-p38 antibodies were purchased from Cell Signaling Technology (1:1,000; Danvers, MA, USA).

\section{Enzyme-linked Immunosorbent Assay (ELISA)}

The concentration of inflammatory cytokines (IL-1 $\beta$, IL-6, and IL-8) and extracellular matrix (ECM)catabolic enzymes (MMP-1, MMP-3, and MMP-13) in the CM, induced by $48 \mathrm{~h}$ of combined-hResistin and IL-1 $\beta$ stimulation or mono-IL-1 $\beta$, was determined by using the commercially available ELISA kits (R\&D Systems) in accordance with the manufacturer's procedure.

\section{Treatment of hIVD cells with IL-1 receptor, p38 MAPK, and p65 NF-kB-specific inhibitors, followed by combined- hResistin and IL-1 $\beta$ stimulation}

hAF and hNP cells were cultured in 6-well plates and incubated at $37^{\circ} \mathrm{C}$ with the IL-1 receptor antagonist IL-1Ra (100 ng/mL), the p38 inhibitor SB203580 (10 uM, Sigma Chemical Co., St. Louis, MO, USA), and IKB kinase inhibitor Bay 11-7082 (5 mM, Sigma) at the indicated concentrations for $1 \mathrm{~h}$ before combinedhResistin and IL-1 $\beta$ stimulation. 


\section{Statistical Analysis}

To obtain the mean \pm standard error of the analysis results of individual cells, six experiments with stimulation of combined-hResistin and IL-1 $\beta$ or mono-IL-1 $\beta$ were conducted. The obtained results were subjected to the analysis of variance with Bonferroni correction post hoc test, as required. $p<0.05$ was considered as statistically significant. All statistical analyses were performed using SPSS software (ver. 21.3, SPSS Inc., Chicago, IL, USA).

\section{Results}

\section{Determination of optimum concentration of hResistin stimulation on hIVD cells In Vitro}

As shown in Fig. 2, in order to investigate the optimal concentration of hResistin treatment on the cultured hAF and hNP cells in vitro, the viability, cytotoxicity and fluorescence image of cytotoxicity of the precultured hAF and hNP cells with 6 different concentrations of hResistin treatment, which are $0,25,50$, 100,200 , and $400 \mathrm{ng} / \mathrm{mL}$, were measured by using WST-1, LDH, and live/dead assay, respectively. The viability and cytotoxicity were measured in mean \pm SEM, whereas the fluorescence image of cytotoxicity was shown in $200 \mu \mathrm{m}$ scale bar. Based on the resultant fluorescence image of cytotoxicity shown in Fig. 2(C), $100 \mathrm{ng} / \mathrm{ml}$ of hResistin was selected as an optimal concentration in this study since the dead hAF and hNP cells began to be observed in the group with $200 \mathrm{ng} / \mathrm{ml}$ of hResistin stimulation. This can be also supported by the significant increase of secreted LDH level of hAF and hNP cells as shown in Fig. 2(B), without a significant decrease of their viability of the same group as shown in Fig. 2(A), with respect to that of the naïve group.

\section{Correlation between hResistin stimulation and phosphorylation of p38 MAPK and p65 NF-KB}

As shown in Fig. 3, the signaling pathway and the densitometric quantification of phosphorylation p38 MAPK and p65 NF-KB induced by five-different durations of hResistin stimulation $(0,15,30,60$, and 120 min) on hAF and hNP cells, which was carried out to investigate the correlation between the correlation between hResistin stimulation and the phosphorylation p38 MAPK and p65 NF-kB signaling pathways, the largest relative fold-change of p38 MAPK and p65 NF-KB phosphorylation induced by 30 and 120 min of hResistin stimulation on hAF cells were determined to $2.18 \pm 0.33,1.6 \pm 0.12$, respectively. Whereas, for hNP cells stimulated with 120 min of hResistin, they were determined to be $1.33 \pm 0.05$, and $1.24 \pm 0.02$, respectively.

\section{Gene expression of inflammasome components}

The mRNA expression of inflammasome components, such as nod-like receptor protein 3 (NLRP3), Caspase-1, and pro-IL-1 $\beta$, was induced by hResistin stimulation, and the results are shown in Fig. 4. The 
relative fold-changes of NLRP3, Caspase-1, and pro-IL1 $\beta$ gene expression compared to those in the unstimulated and control groups were $2.87 \pm 0.36,0.82 \pm 0.08$, and $1.44 \pm 0.01$ in hAF cells and $3.47 \pm$ $1.18,1.56 \pm 0.14$, and $2.87 \pm 0.80$ in hNP cells, respectively (Fig. $4(A)$ and (B)). Gene expression of inflammasome components induced by hResistin stimulation was higher in hNP cells than in hAF cells, and caspase- 1 was not expressed in hAF cells.

\section{The role of stimulation of hResistin on the promotion of IL- $1 \beta$-associated pro-inflammatory cytokine cascade activation}

As shown in Figs. 5 and 6 , the change in secretion level of inflammatory mediators (IL-1 1 , IL-6, IL-8, MMP1, MMP-3, and MMP-13) induced by two different stimulation of combined-hResistin and IL-1 $\beta$ or monoIL-1 $\beta$ for $48 \mathrm{~h}$ on both $\mathrm{hAF}$ and $\mathrm{hNP}$, measured via ELISA, the mean optical density change of inflammatory mediators induced by the combined-hResistin and IL-1 $\beta$ stimulation on hAF cells, which was determined to be $0.60 \pm 0.01,7.47 \pm 0.29,9.96 \pm 0.33,4.49 \pm 0.78,191.48 \pm 16.14$, and $0.44 \pm 0.02$ $\mathrm{ng} / \mathrm{mL}$ respectively, was observed to be significantly greater than that of the same induced by mono-IL-1 $\beta$ stimulation which was determined to be $0.17 \pm 0.004,0.77 \pm 0.23,5.62 \pm 1.39,1.19 \pm 0.39,70.87 \pm 8.90$, and $0.06 \pm 0.01 \mathrm{ng} / \mathrm{mL}$, respectively. Similarly, in the case of the mean optical density change of IL-1 $\beta$, IL6, IL-8, MMP-1, MMP-3, and MMP-13 induced by the combined-hResistin and IL-1 $\beta$ stimulation on hNP cells, which was determined to be $0.64 \pm 0.01,7.54 \pm 1.57,27.43 \pm 2.19,3.60 \pm 0.69,254.40 \pm 44.66$, and $0.57 \pm 0.03 \mathrm{ng} / \mathrm{mL}$, respectively, was also found to be significantly greater than that of the same induced by mono-IL-1 $\beta$ stimulation which was determined to be $0.24 \pm 0.02,0.75 \pm 0.19,8.85 \pm 2.06,1.43 \pm 0.50$, $115.74 \pm 22.31$, and $0.21 \pm 0.02 \mathrm{ng} / \mathrm{mL}$, respectively.

\section{Role of hResistin stimulation-induced upregulation of IL-1 $\beta$, p38 MAPK, and p65 NF-KB and the combined hResistin and IL-1 $\beta$ stimulation-induced IL-6, IL-8, MMP-1, MMP-3, and MMP-13 changes via stimulation with IL-1 $\beta$, p38 MAPK, and p65 NF-kB specific inhibitors}

To confirm the pro-inflammatory cytokine activation nature of hResistin, the effects of IL-1 3, p38 MAPK, and p65 NF-KB on the upregulation of inflammatory mediators induced by the combined-hResistin and IL$1 \beta$ stimulation and the reduction in secretion of inflammatory mediators induced by the combinedhResistin and IL-1 $\beta$ stimulation and attributed to the treatment with IL-1, p38 MAPK, and p65 NF-KBspecific inhibitors IL-1Ra, SB203580, and BAY 11-7082, respectively, were analyzed compared to those observed upon the combined- hResistin and IL-1 $\beta$ or mono-IL-1 $\beta$ stimulation. As shown in Figs. 7 and 8 , a clear trend between p65 NF-KB and the upregulation of combined-hResistin and IL-1 $\beta$ stimulation induced inflammatory mediators was observed via a significant reduction attributed to the treatment with BAY 117082 in both hAF and hNP cells. Regarding IL-1Ra treatment, the inflammatory cytokine levels were 
similar to those obtained with BAY 11-7082 treatment, and the inflammatory cytokines were inhibited at higher levels than those in the mono-IL-1 $\beta$ stimulation with IL-1Ra treatment in both hAF and hNP cells (Figs. 7 and 8, ( () BAY 11-7082 treatment panels). When correlating IL-1Ra treatment and ECM catabolic enzymes, the inhibition of ECM catabolic enzymes in NP cells was markedly higher than that in hAF cells. In hAF cells, the inhibition levels of mono-IL-1 $\beta$ in the IL-1Ra treatment group were higher than those in the IL-1Ra treatment group (Figs. 7 and 8, (I) IL-1 Ra treatment panels). In the correlation between SB203580 treatment and the inflammatory mediators, increased inflammatory mediator levels in the combined groups tended to decrease almost to the same levels as those observed in the mono-IL-1 $\beta$ stimulation groups (Figs. 7 and 8, (II) SB203580 treatment panels). In addition, mono- IL-1 $\beta$ stimulation with SB203580 treatment showed a higher tendency to decrease than combined stimulation with SB203580 treatment in both hAF and hNP cells.

\section{Discussion}

Although several studies investigated the correlation between hResistin and the pro-inflammatory signaling pathways, the main cause of the promoting effect of hResistin on the promotion of IL-1 $\beta$ associated pro-inflammatory cytokine cascades is still not fully understood. However, it is suspected that the activation of toll-like receptor (TLR)4, which is reported to serve as a receptor for the pro-inflammatory effects of resistin in human cells, could trigger the upregulation of not only the IL-1 family of cytokines but also of TNF-a ${ }^{11-14}$. As shown in Fig. 3, hResistin stimulation of both hAF and hNP cells induced the phosphorylation of p38 MAPK and p65 NF-KB, which promote inflammatory cytokine activation cascades in hIVD tissues. MAPK is activated by various exogenous factors and is involved in various inflammatory diseases, such as osteoarthritis, rheumatoid arthritis, and neurodegenerative disease ${ }^{15,16}$. MAPKs are divided into three subunit types: p38, ERK, and JNK. Regarding the inflammatory cytokines of the hIVD, p38 and ERK are associated with a decrease in ECM synthesis, and p38 and JNK are involved in the activation of ECM catabolic enzymes, such as MMPs ${ }^{17,18}$. Another important pathway involved in the inflammatory response involves NF-kB, a central component of the hIVD cellular response to damage, stress, and inflammation. NF-KB is composed of the most common heterodimer, the p50/p65 subunit complex, which regulates several pro-inflammatory mediators, such as TNF-a, IL-1 $\beta$, IL-6, and MMPs ${ }^{19,20}$. Thus, p38 MAPK and p65 NF-KB may play important roles in inflammatory IVD degeneration. The phosphorylation of p38 MAPK and p65 NF-KB is associated with TLR4-related inflammasome upregulation ${ }^{21-23}$. The interaction between hResistin and hIVD cells results in the mRNA expression of NLRP3, Caspase-1, and pro-IL-1 $\beta$. The inflammasome components involved in the inflammatory response are shown in Fig. 4. The gene expression of hResistin-induced inflammasome components exerts a greater influence in hNP cells, and the role of hResistin in the inflammatory response is expected to be more dominant in hNP cells than in hAF cells. Nevertheless, the gene expression induced by hesistin stimulation was significantly different from that of the unstimulated control groups, but the fold-change value was not significantly different; thus, hResistin mono-stimulation did not seem to significantly affect inflammatory cytokine expression in hIVD cells. 
Based on the significant increase in secretion of IL-1 $\beta$, IL-6, IL-8, MMP-1, MMP-3, and MMP-13 induced by combined-hResistin and IL-1 $\beta$ stimulation of both hAF and hNP cells, compared with that induced by IL$1 \beta$ mono-stimulation (Figs. 5 and 6 ), the promoting effect of hResistin on the activation of the IL-1 $\beta$ associated pro-inflammatory cytokine cascade was observed. This cascade is associated with the progression of obesity-related inflammatory disc degeneration; this further supports the low-grade proinflammatory cytokine cascade activation nature of hResistin, which is widely reported to be associated with the development of insulin resistance in obesity 2,6,24-27. hResistin is primarily expressed in immune cells, such as monocytic cells, which are key players in the inflammatory response, in contrast to rodent resistin, which releases rodent adipocytes in white adipose tissues ${ }^{13,14,28}$. Thus, hResistin is expected to have a greater influence on the inflammatory response in adipokine-related diseases than rodent resistin. The promoting effect of hResistin on the IL-1 $\beta$-associated pro-inflammatory cytokine cascade might be influenced by the correlation between TLR 4 and hResistin-induced expression of IL-1 $\beta$ and the phosphorylation of p38 MAPK and p65 NF-KB. The treatment with each specific inhibitor of IL-1 $\beta$, p38 MAPK, and p65 NF-KB effectively inhibited the promoting effect of hResistin-related inflammatory mediator protein expression (Figs. 7 and 8). The IL-1 signaling inhibition of the combined groups reduced both the increased inflammatory cytokine and ECM catabolic enzyme expression levels, which may be due to a promoting effect of hResistin-induced low-grade inflammatory cytokine activation in the IL-1 $\beta$ associated inflammatory response. Nevertheless, the decrease in levels of ECM catabolic enzymes in hAF cells was lower than that in hNP cells, which is expected to be more associated with hNP cells than with hAF cells in the correlation between hResistin and IL-1 $\beta$-associated inflammatory response. When comparing p38 MAPK and p65 NF-KB, which are involved in the expression of inflammatory mediators in hIVDD, the NF-KB signaling inhibition showed a greater reduction in levels of inflammatory mediators than p38 MAPK signaling inhibition, indicating that NF-KB signaling plays a more important role than p38 MAPK signaling. It is also thought that pathways other than the p38 MAPK pathway may be involved in ECM catabolic enzyme expression ${ }^{29,30}$. In 2014, Eltom et al. reported the release of IL-1 $\beta$, induced by TLR4, which was activated via direct exposure to endotoxin lipopolysaccharide (LPS) in living mouse lung tissues in vivo ${ }^{31}$. Similarly, in the following year, Lin et al. also reported an increased expression of IL-1 $\beta$ and TNF- $\alpha$ induced by the activation of TLR4, which was induced by in vitro stimulation of synovial fibroblast cells with LPS ${ }^{32}$. It is widely accepted that IVDD is evident by the increased secretion of TNF, IL-1a, IL-1 $\beta$, IL-6, IL-17, IL-8, and IL-2, which leads to an imbalance in catabolic and anabolic responses, leading to the degeneration of IVD tissues, as well as disc herniation and radicular pain via the promotion of extracellular matrix degradation, chemokine secretion, and IVD cell phenotype changes ${ }^{33-35}$. However, increases in the expression of MMP-1, MMP-3, MMP-7, MMP-9, and MMP-13 are associated with the promotion of a degenerative response ${ }^{30,36-38}$. However, the specific inflammatory disc degenerationassociated inflammatory pathways that are responsible for the promoting effect of hResistin on the promotion of IL-1 $\beta$-associated inflammatory cytokine cascade activation remain to be identified and should be investigated in future studies.

Additionally, it was interesting to observe that the changes in secretion levels of all inflammatory mediators in hNP cells induced by the combined-hResistin and IL-1 $\beta$ stimulation were determined to be 
marginally greater than those induced in hAF cells. This trend may be due to the accelerated dehydration and desiccation of NP cells, induced by the promotion of apoptosis of NP cells activated by the IL family of inflammatory cytokines. Wang et al. reported that IL-2, which is associated with the onset of thinning and tearing of hAF cells at the early stage of inflammatory IVDD, promotes the apoptosis of NP cells by upregulating destructive enzymes, such as MMPs or ADAMTS, downregulating the aggrecan expression levels, and altering the levels of collagen type I-II via the death receptor pathway activated by an increased activity of caspase- 3 and caspase-8, which is associated with the upregulation of Fas protein expression ${ }^{39}$. The NP is encapsulated by endplates and the AF has a significant influence on the overall function and homeostasis of the intervertebral discs by acting as a pump to regulate the flow of liquids and gases in the discs via proteoglycan-rich ECM, maintaining the hydration levels ${ }^{40}$.

In conclusion, based on the significant upregulation of target inflammatory IVDD-associated inflammatory mediators, induced by the combined-hResistin and IL-1 $\beta$ stimulation of hIVD cells, compared to that induced by mono-IL-1 $\beta$ stimulation, the promoting effect of hResistin, which is associated with the development of insulin resistance in obesity, on the progression of inflammatory IVDD via upregulation of inflammatory mediators associated with the activation of IL-1 $\beta$ proinflammatory cytokine cascade has been observed. These results further support the low-grade proinflammatory cytokine cascade activation nature of hResistin. Moreover, the significance of NP in the progression of inflammatory IVDD has been observed via a marginally greater secretion of inflammatory mediators from NP cells than from AF cells, after stimulation with hResistin and IL-1 $\beta$. The results of our study improve our understanding of the pro-inflammatory mechanism of action of hResistin, which is associated not only with the progression of inflammatory IVDD but also with other obesity-and diabetesrelated inflammatory diseases.

\section{Declarations}

\section{Author contributions}

Jae Hee Shin designed and performed the experiments. Jae Hee Shin, SeongHyun Park, Hansang Cho, Joo Han Kim and Hyuk Choi contributed to the writing of the manuscript and analyzed the data. All authors reviewed the manuscript.

\section{Acknowledgements}

This study protocols were approved by the Institutional Review Board of the Korea University College of Medicine. This research was supported by the Basic Science Research Program through the National Research Foundation of Korea (NRF) funded by the Ministry of Education (2020R1F1A1068910).

\section{References}


1. Hotamisligil, G. S., Shargill, N. S. \& Spiegelman, B. M. Adipose expression of tumor necrosis factor-a: Direct role in obesity-linked insulin resistance. Science 259, 87-91 (1993).

2. Qiu, W. et al. Resistin increases platelet P-selectin levels via p38 MAPK signal pathway. Diabetes and Vascular Disease Research 11, (2014).

3. Silswal, N. et al. Human resistin stimulates the pro-inflammatory cytokines TNF-a and IL-12 in macrophages by NF-kB-dependent pathway. Biochemical and Biophysical Research Communications 334, (2005).

4. Li, X. et al. Intervertebral disc degeneration in mice with type II diabetes induced by leptin receptor deficiency. BMC Musculoskeletal Disorders 21, 1-10 (2020).

5. Ding, W. et al. Leptin induces terminal differentiation of rat annulus fibrosus cells via activation of MAPK signaling. Anatomical Record 296, 1806-1812 (2013).

6. Ruiz-Fernández, C. et al. Molecular relationships among obesity, inflammation and intervertebral disc degeneration: Are adipokines the common link? International Journal of Molecular Sciences 20, (2019).

7. Sharma, A. The Role of Adipokines in Intervertebral Disc Degeneration. Medical Sciences 6, 34 (2018).

8. Segar, A. H., Fairbank, J. C. T. \& Urban, J. Leptin and the intervertebral disc: a biochemical link exists between obesity, intervertebral disc degeneration and low back pain-an in vitro study in a bovine model. European Spine Journal 28, 214-223 (2019).

9. Liu, C. et al. Resistin promotes intervertebral disc degeneration by upregulation of ADAMTS-5 through p38 MAPK signaling pathway. Spine 41, (2016).

10. Seki, S. et al. Effect of small interference RNA (siRNA) for ADAMTS5 on intervertebral disc degeneration in the rabbit anular needle-puncture model. Arthritis Research and Therapy 11, 1-10 (2009).

11. Li, Z. et al. Resistin promotes CCL4 expression through toll-like receptor-4 and activation of the p38MAPK and NF-KB signaling pathways: implications for intervertebral disc degeneration. Osteoarthritis and Cartilage 25, 341-350 (2017).

12. Tarkowski, A., Bjersing, J., Shestakov, A. \& Bokarewa, M. I. Resistin competes with lipopolysaccharide for binding to toll-like receptor 4. Journal of Cellular and Molecular Medicine 14, 1419-1431 (2010).

13. al Hannan, F. \& Culligan, K. G. Human resistin and the RELM of Inflammation in diabesity. Diabetology and Metabolic Syndrome 7, 1-11 (2015).

14. Azamar-Llamas, D., Hernández-Molina, G., Ramos-Ávalos, B. \& Furuzawa-Carballeda, J. Adipokine Contribution to the Pathogenesis of Osteoarthritis. Mediators of Inflammation 2017, (2017).

15. Lawrence, M. C. et al. The roles of MAPKs in disease. Cell Research 18, 436-442 (2008).

16. Kim, E. K. \& Choi, E. J. Compromised MAPK signaling in human diseases: an update. Archives of Toxicology 89, 867-882 (2015). 
17. Wuertz, K., Vo, N., Kletsas, D. \& Boos, N. Inflammatory and catabolic signalling in intervertebral discs: The roles of NF-KB and map kinases. European Cells and Materials 23, 103-120 (2012).

18. Zhang, X. et al. Obesity Mediates Apoptosis and Extracellular Matrix Metabolic Imbalances via MAPK Pathway Activation in Intervertebral Disk Degeneration. Frontiers in Physiology 10, 1-11 (2019).

19. Sun, Z., Yin, Z., Liu, C. \& Tian, J. The Changes in the Expression of NF-KB in a Degenerative Human Intervertebral Disc model. Cell Biochemistry and Biophysics 72, 115-122 (2015).

20. Zhongyi, S., Sai, Z., Chao, L. \& Jiwei, T. Effects of nuclear factor kappa B signaling pathway in human intervertebral disc degeneration. Spine 40, 224-232 (2015).

21. Boaru, S. G. et al. NLRP3 inflammasome expression is driven by NF-KB in cultured hepatocytes. Biochemical and Biophysical Research Communications 458, 700-706 (2015).

22. Li, D., Ren, W., Jiang, Z. \& Zhu, L. Regulation of the NLRP3 inflammasome and macrophage pyroptosis by the p38 MAPK signaling pathway in a mouse model of acute lung injury. Molecular Medicine Reports 18, 4399-4409 (2018).

23. Schroder, K. \& Tschopp, J. The Inflammasomes. Cell 140, 821-832 (2010).

24. Lee, J. H. et al. Resistin is elevated following traumatic joint injury and causes matrix degradation and release of inflammatory cytokines from articular cartilage in vitro. Osteoarthritis and Cartilage $17,613-620$ (2009).

25. Dasari \& Raghunath, V. Obesity and Type II diabetes mellitus: Is resistin the link? Journal of Diabetes and Endocrine Practice 1, 1 (2018).

26. Choi, S. H., Hong, E. S. \& Lim, S. Clinical implications of adipocytokines and newly emerging metabolic factors with relation to insulin resistance and cardiovascular health. Frontiers in Endocrinology 4, 1-7 (2013).

27. Gómez, R. et al. What's new in our understanding of the role of adipokines in rheumatic diseases? Nature Reviews Rheumatology 7, 528-536 (2011).

28. Mancuso, P. The role of adipokines in chronic inflammation. ImmunoTargets and Therapy vol. 5 4756 (2016).

29. Miao, D. \& Zhang, L. Leptin modulates the expression of catabolic genes in rat nucleus pulposus cells through the mitogen-activated protein kinase and Janus kinase $2 /$ signal transducer and activator of transcription 3 pathways. Molecular medicine reports 12, 1761-1768 (2015).

30. Khokha, R., Murthy, A. \& Weiss, A. Metalloproteinases and their natural inhibitors in inflammation and immunity. Nature Reviews Immunology 13, 649-665 (2013).

31. Eltom, S. et al. TLR4 activation induces IL-1 $\beta$ release via an IPAF dependent but caspase $1 / 11 / 8$ independent pathway in the lung. Respiratory Research 15, 1-12 (2014).

32. Lin, X., Kong, J., Wu, Q., Yang, Y. \& Ji, P. Effect of TLR4/MyD88 signaling pathway on expression of IL-1 $\beta$ and TNF- $\alpha$ in synovial fibroblasts from temporomandibular joint exposed to lipopolysaccharide. Mediators of Inflammation 2015, (2015). 
33. Risbud, M. v. \& Shapiro, I. M. Role of cytokines in intervertebral disc degeneration: Pain and disc content. Nature Reviews Rheumatology 10, (2014).

34. Park, J. J. et al. Induction of proinflammatory cytokine production in intervertebral disc cells by macrophage-like THP-1 cells requires mitogen-activated protein kinase activity. Journal of Neurosurgery: Spine 24, 167-175 (2016).

35. Molinos, M. et al. Inflammation in intervertebral disc degeneration and regeneration. Journal of the Royal Society Interface 12, (2015).

36. Zigouris, A., Alexiou, G. A., Batistatou, A., Voulgaris, S. \& Kyritsis, A. P. The role of matrix metalloproteinase 9 in intervertebral disc degeneration. Journal of Clinical Neuroscience 18, $1424-$ 1425 (2011).

37. Roberts, S. et al. Matrix metalloproteinases and aggrecanase: Their role in disorders of the human intervertebral disc. Spine 25, 3005-3013 (2000).

38. Vo, N. v. et al. Expression and regulation of metalloproteinases and their inhibitors in intervertebral disc aging and degeneration. Spine Journa/ 13, 331-341 (2013).

39. Wang, Z., Wang, G., Zhu, X., Geng, D. \& Yang, H. Interleukin-2 is upregulated in patients with a prolapsed lumbar intervertebral disc and modulates cell proliferation, apoptosis and extracellular matrix metabolism of human nucleus pulposus cells. Experimental and Therapeutic Medicine 10, 2437-2443 (2015).

40. Biyani, A. \& Andersson, G. B. J. Low back pain: pathophysiology and management. The Journal of the American Academy of Orthopaedic Surgeons 12, 106-115 (2004).

\section{Figures}

(A) IVD cells isolation from patient

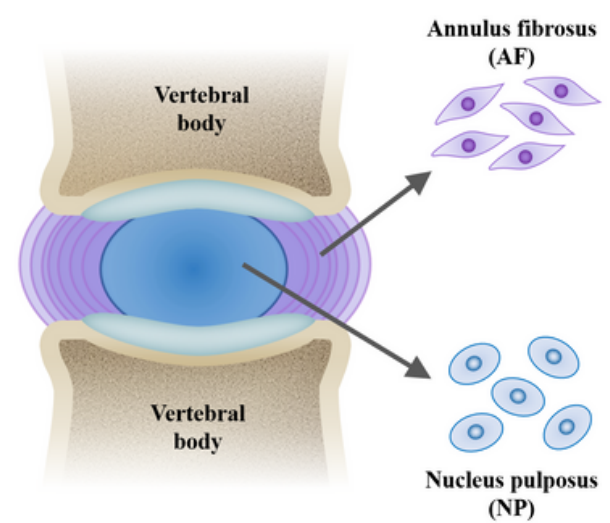

Naïve IVD cells

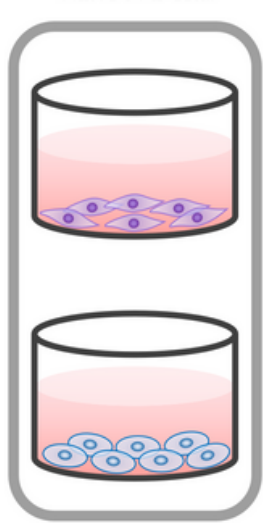

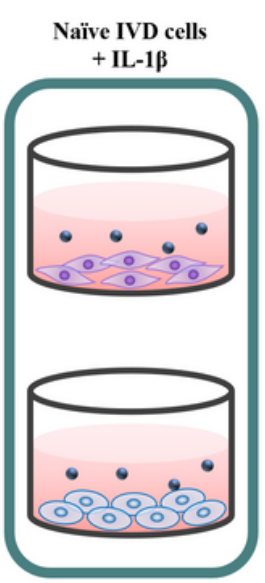

(B)

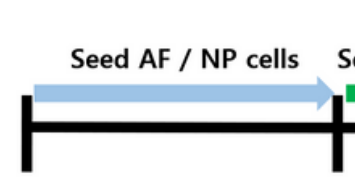

Day (hour) -1 (-24 h)
0
Settlement / stabilization Serum-free starvation

Stimulate combined- hResistin and IL-1 $\beta$ / mono-IL-1 $\beta$

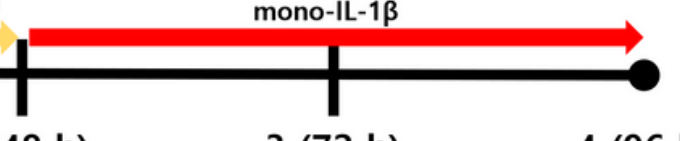


Figure 1

(A) Schematic design and (B) timeline of the experiment used for the combined-hResistin and IL-1 $\beta$ or mono-IL-1 $\beta$ stimulation of hAF and hNP.

(A)

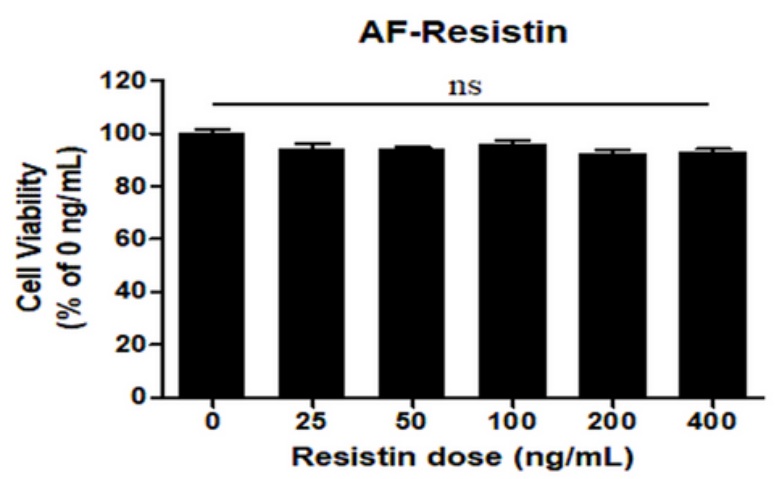

(B)

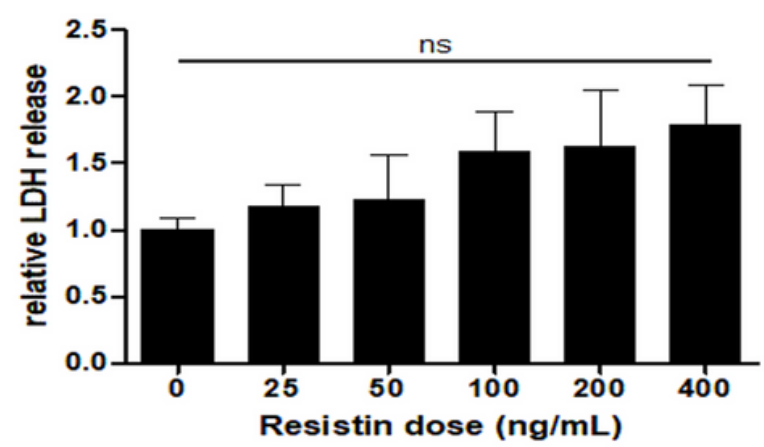

NP-Resistin

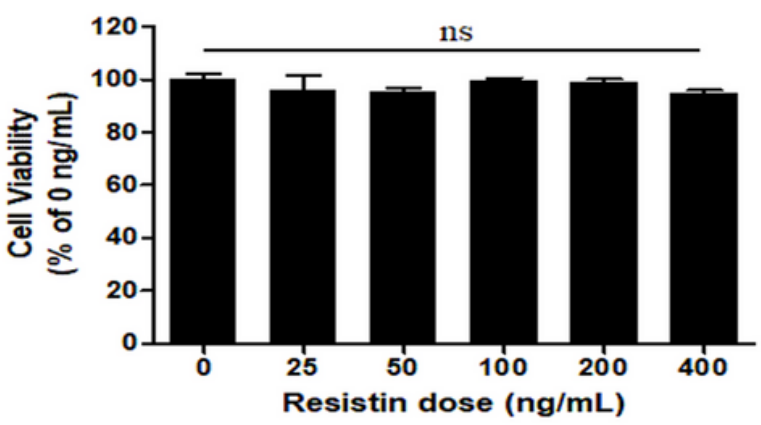

NP-Resistin

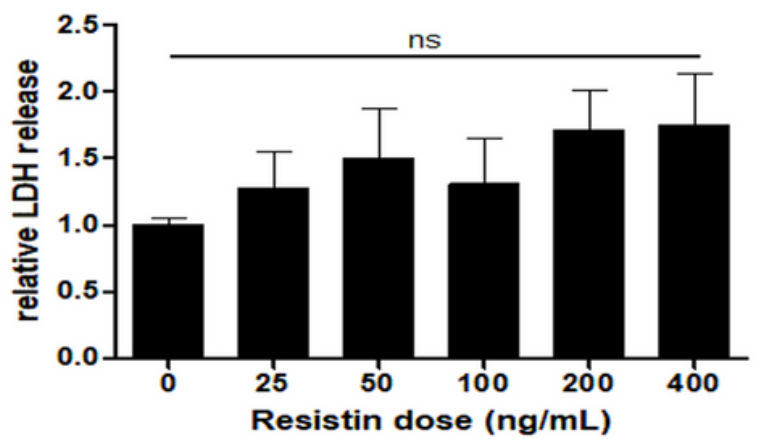

(C) hAF cells with Resistin concentration

Dead cells

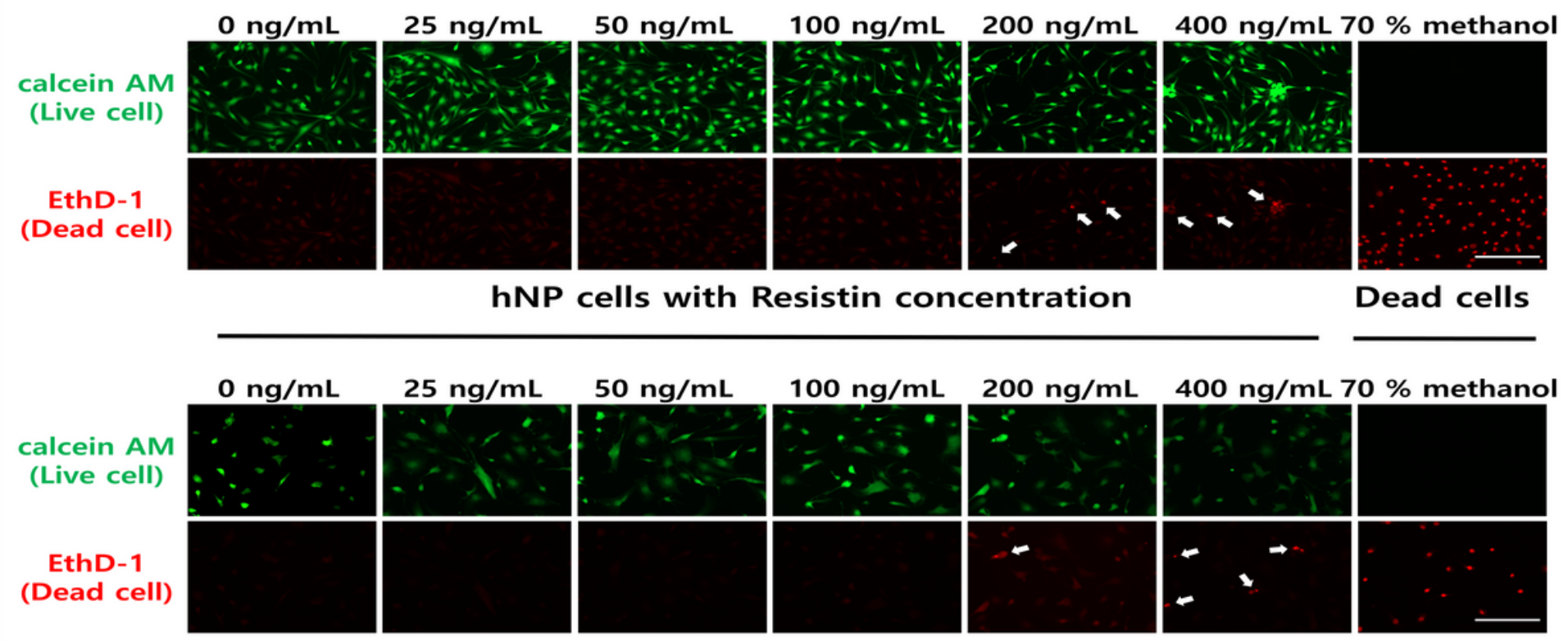

Figure 2

The mean \pm SEM of (A) viability, (B) cytotoxicity, and (C) fluorescence image (scale bar $=200 \mu \mathrm{m}$ ) of the live/dead assay of hIVD cells stimulated with six different concentrations of hResistin $(0,25,50,100,200$ 
(A) Resistin p-p38 p38 p-p65 p65 $\beta$-actin
AF with

\section{mins}
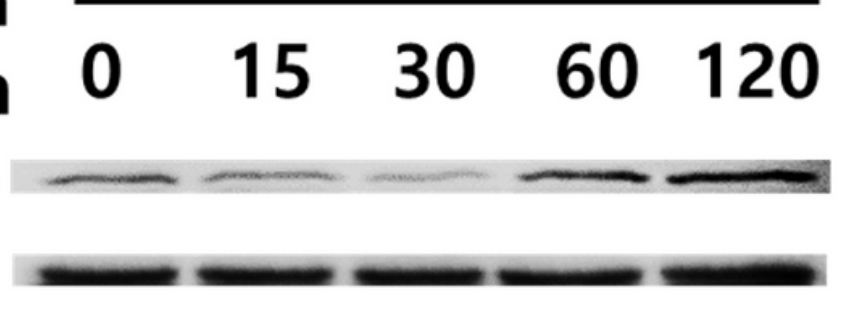

(C)

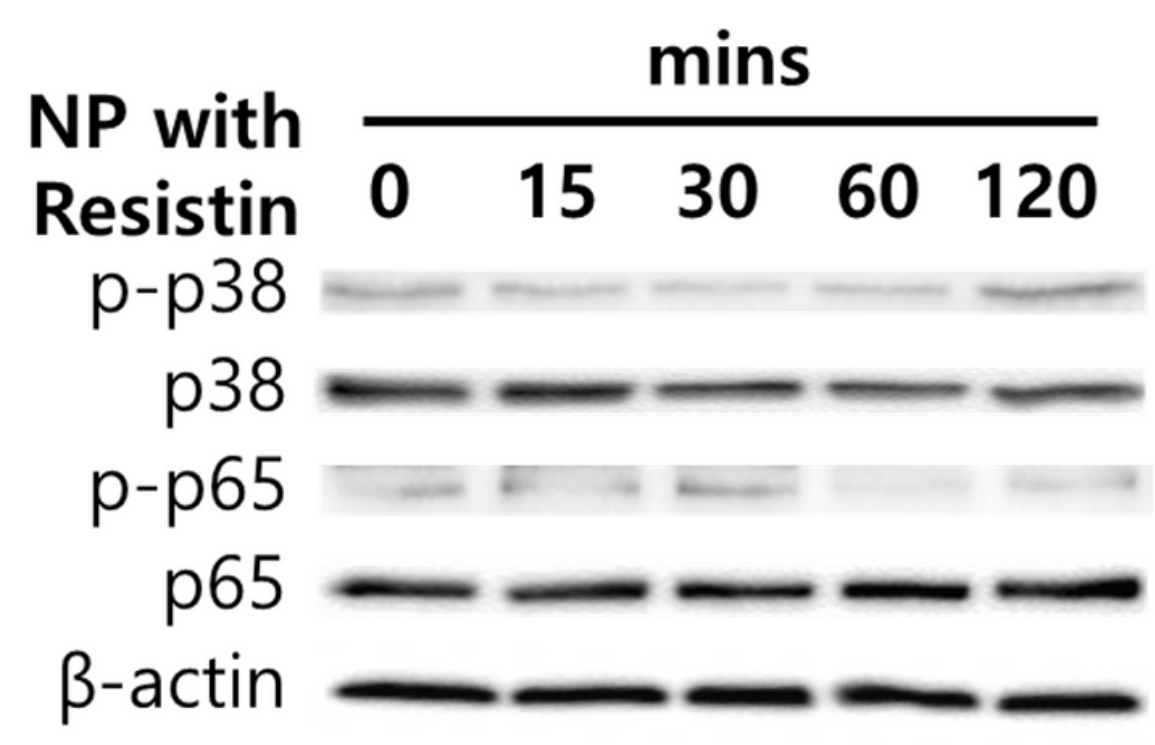

(B)
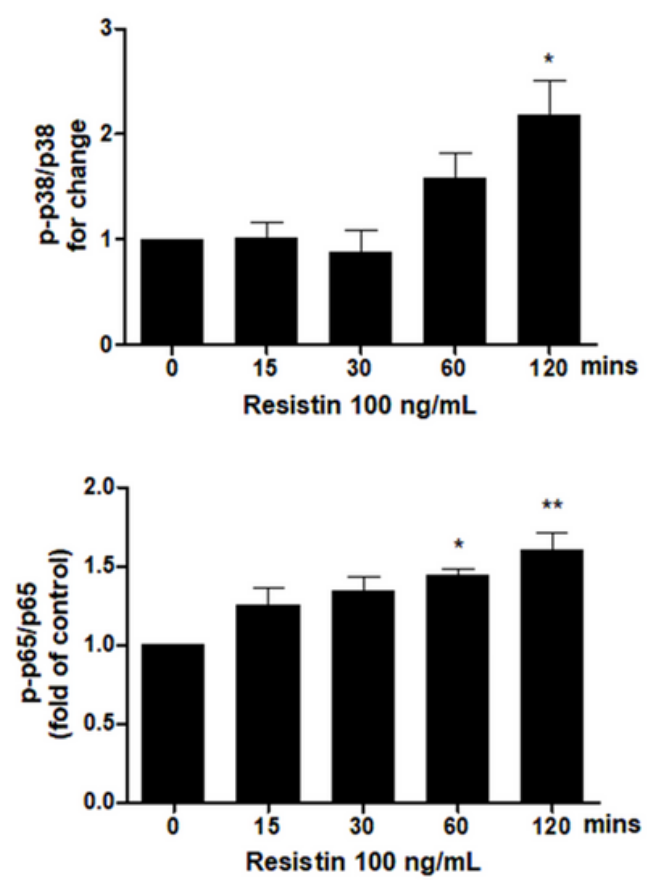

(D)
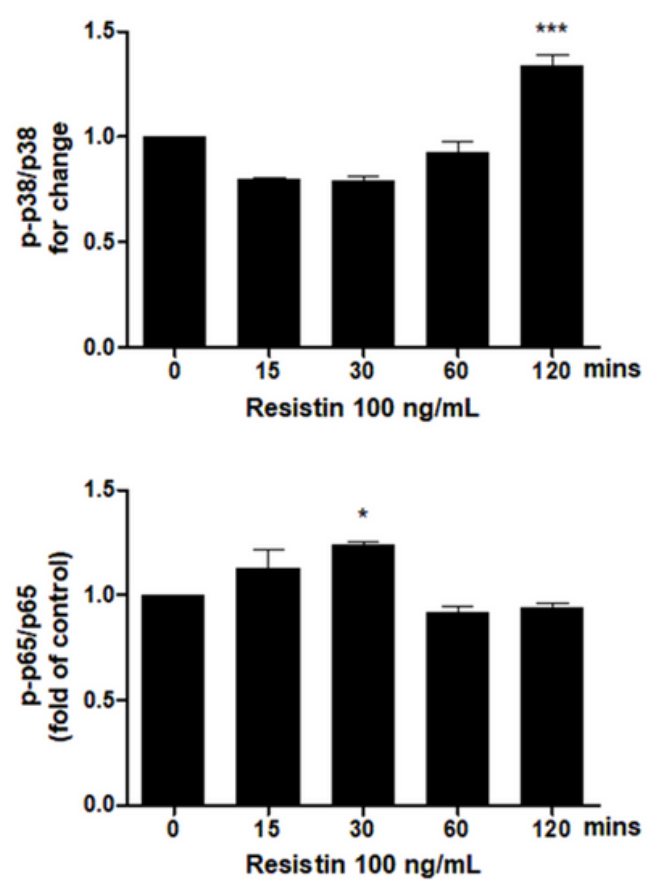

Figure 3

(A) and (C) The signaling pathway and (B) and (D) the densitometric quantification $\left({ }^{\star} p<0.05\right.$, $* \star p<$ 0.01 , and $* \star \star p<0.001$ ) of phosphorylated p38 MAPK and p65 NF-kB induced by 5 different durations of post-hResistin stimulation, compared to the unstimulated naïve control group. 
(A)

AF-Resistin

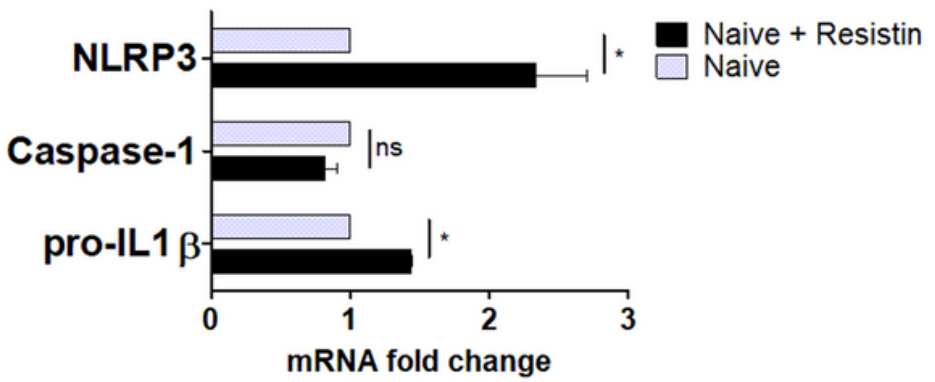

(B) NP-Resistin

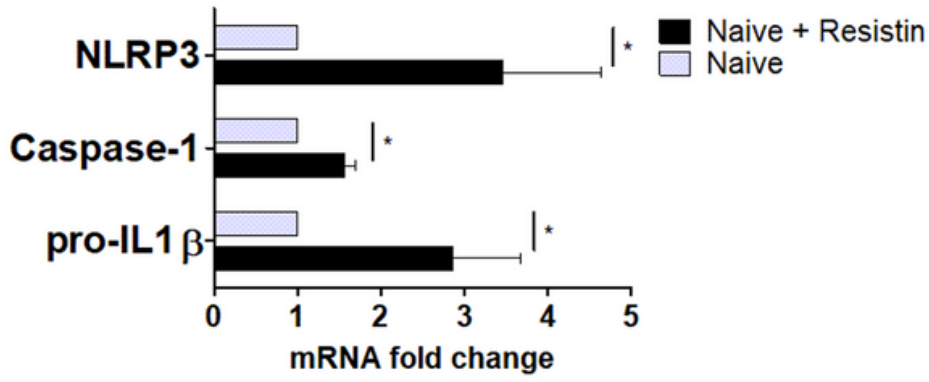

Figure 4

mRNA gene expression of the inflammatory mediators of NLRP3, Caspase-1, and pro-IL $1 \beta$ between IVD cells and hResistin stimulation, compared to unstimulated naïve IVD control groups ( ${ }^{*} p<0.05$ ).

(A)

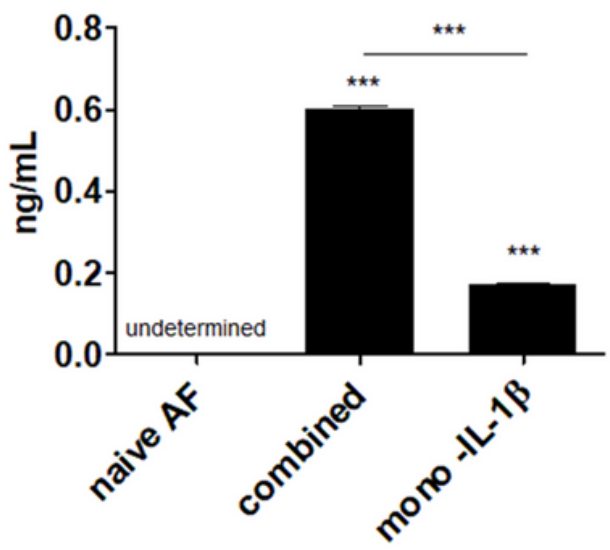

(D)

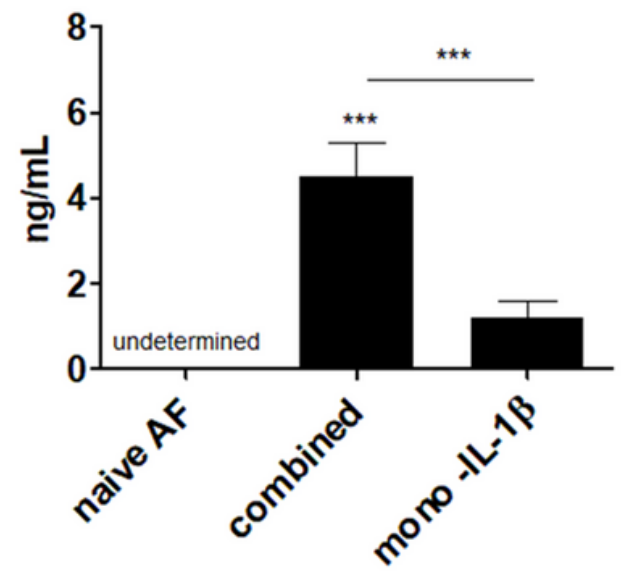

(B)

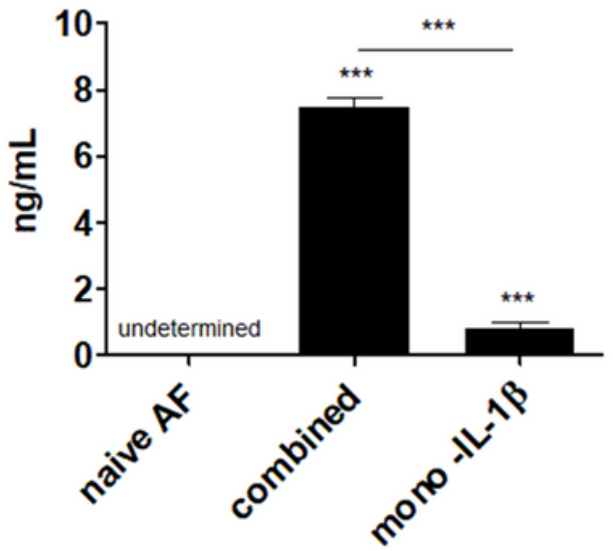

(E)

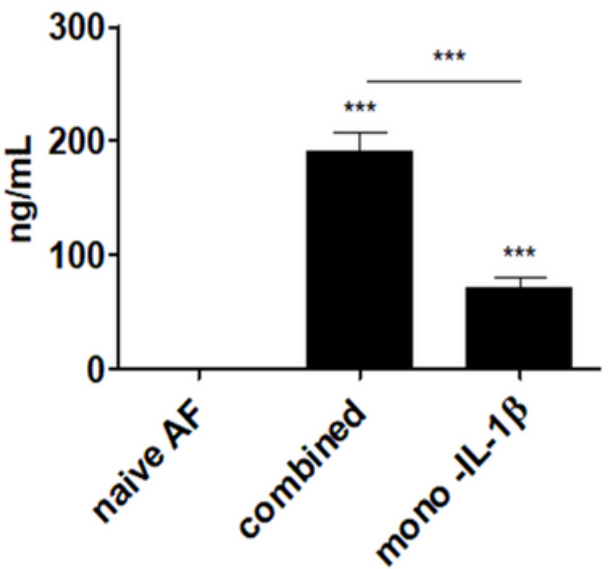

(C)

IL-8

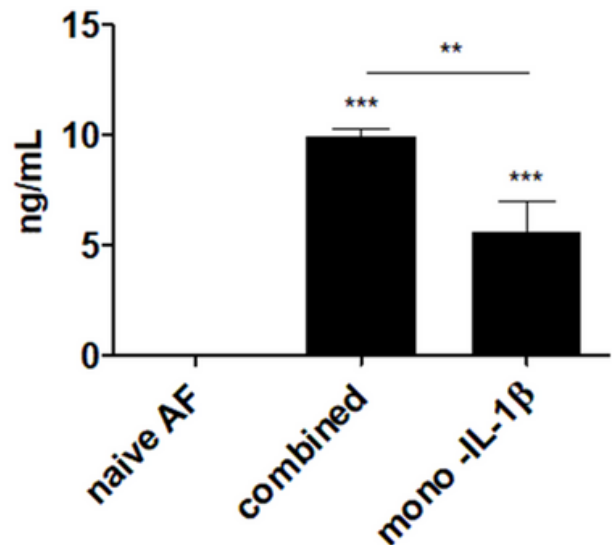

(F)

MMP-13

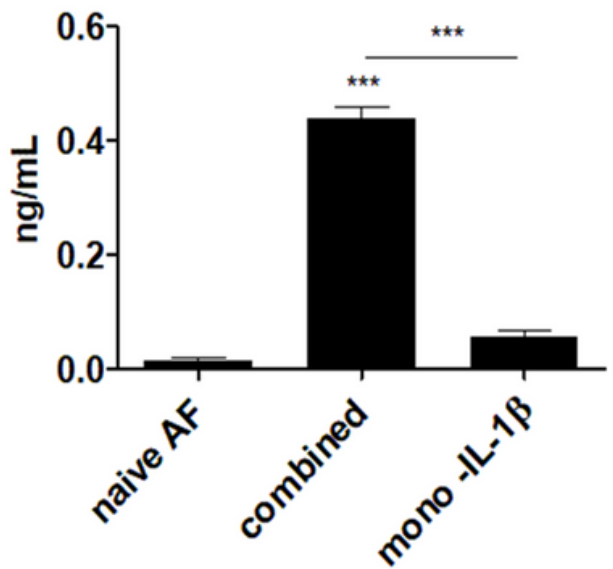

Figure 5 
The change in secretion of (A) IL-1 $\beta$, (B) IL-6, (C) IL-8, (D) MMP-1, (E) MMP-3, and (F) MMP-13 induced by hAF cells stimulated with combined-hResistin and IL-1 $\beta$ or mono-IL-1 $\beta$ for $48 \mathrm{~h}$ with respect to that of naïve group $(* * p<0.005, * * * p<0.001)$.

(A)

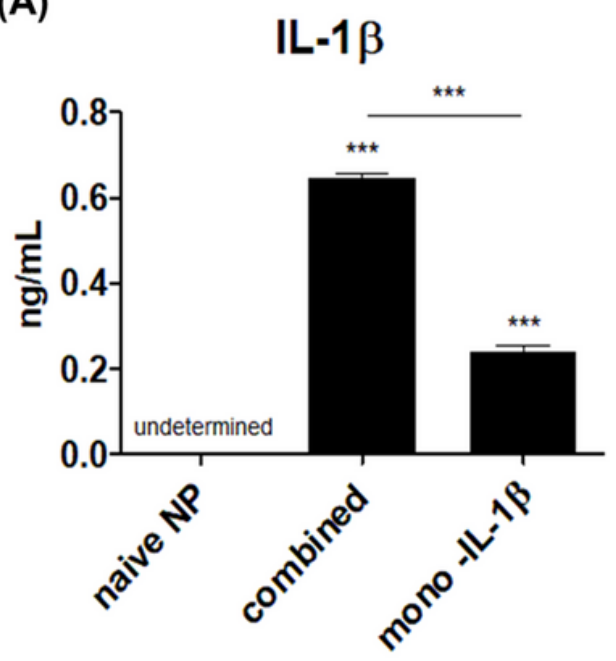

(D)

MMP-1

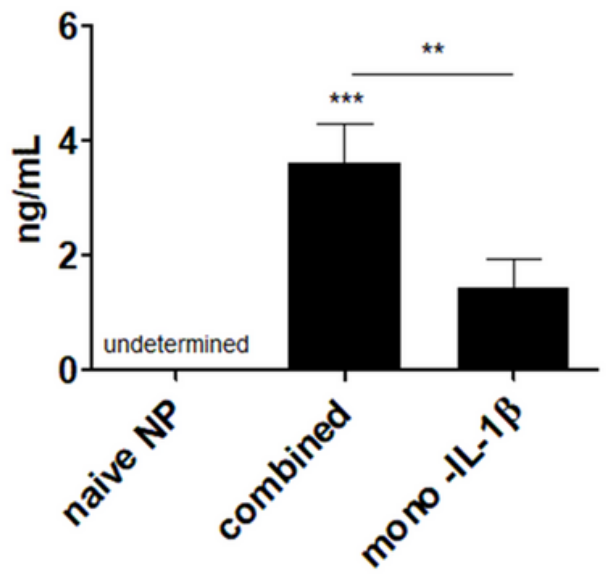

(B)

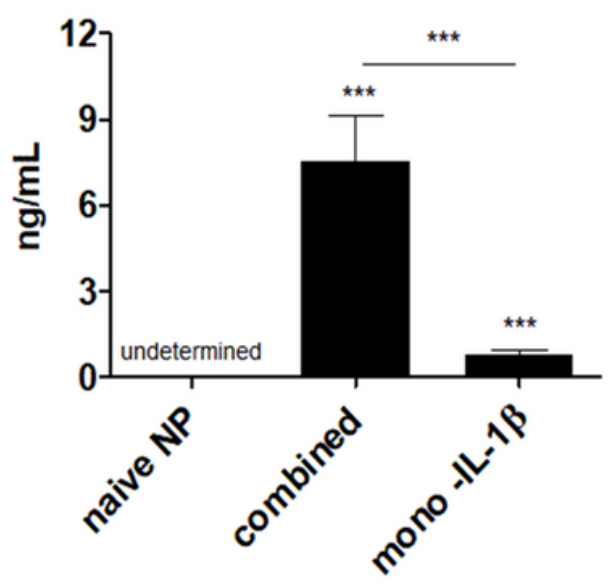

(E)

MMP-3

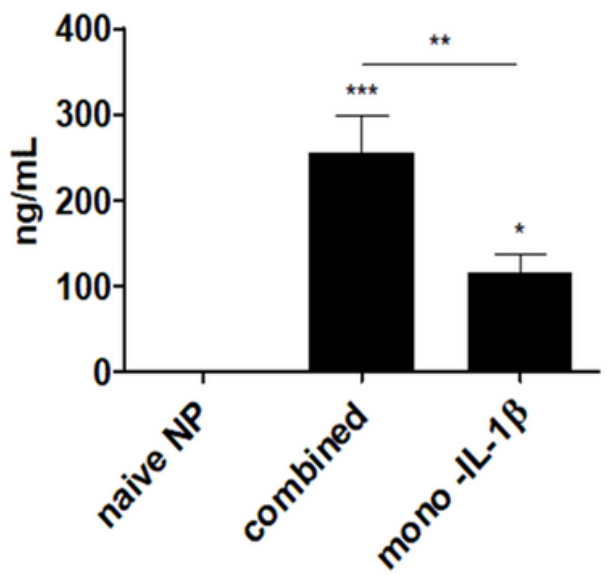

(C)

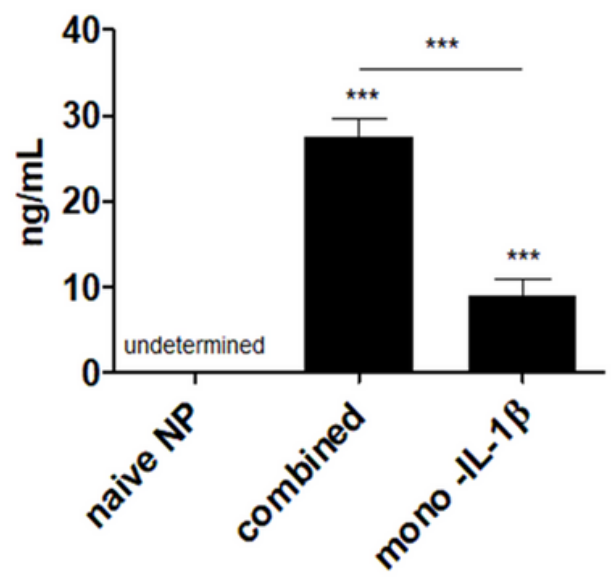

(F)

MMP-13

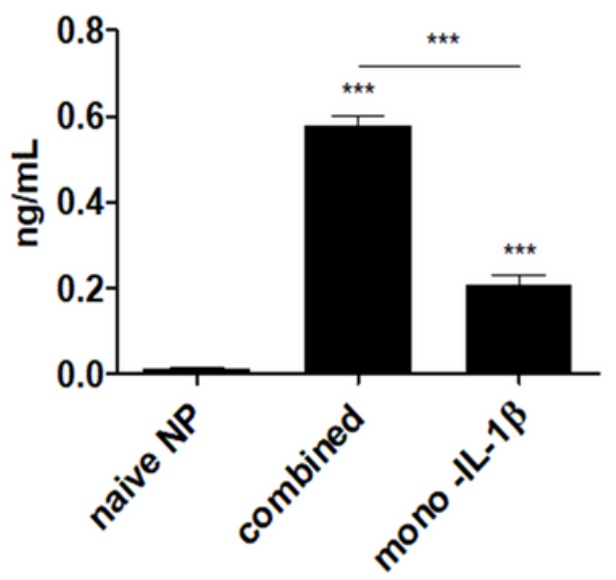

Figure 6

The change in secretion of (A) IL-1 $\beta,(B) I L-6,(C)$ IL-8, (D) MMP-1, (E) MMP-3, and (F) MMP-13 induced by hNP cells stimulated with combined-hResistin and IL-1 $\beta$ or mono-IL-1 $\beta$ for $48 \mathrm{~h}$ with respect to that of naïve group $\left({ }^{\star} p<0.05,{ }^{\star *} p<0.005,{ }^{\star * \star} p<0.001\right)$. 
(I) hAF - IL-1Ra

(A)

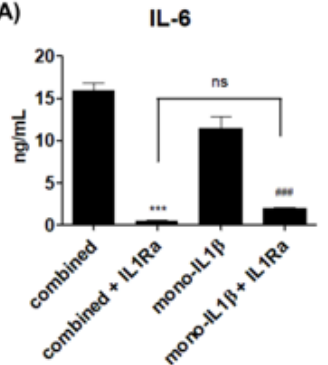

(B)

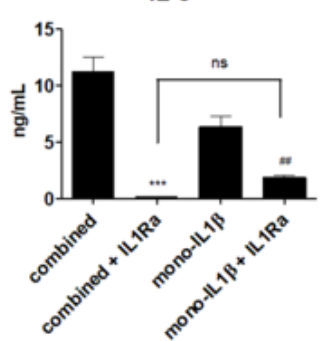

(C)

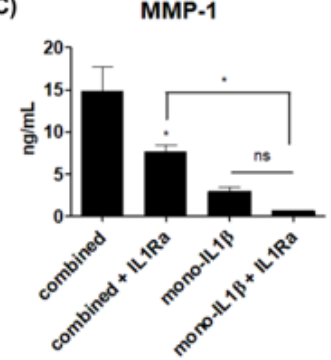

(D)

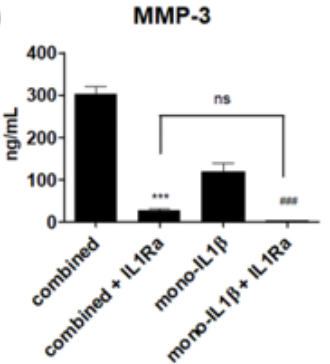

(E)

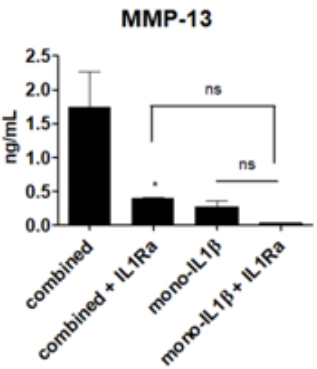

(II) hAF - SB203580

(III) hAF - BAY 11-7082
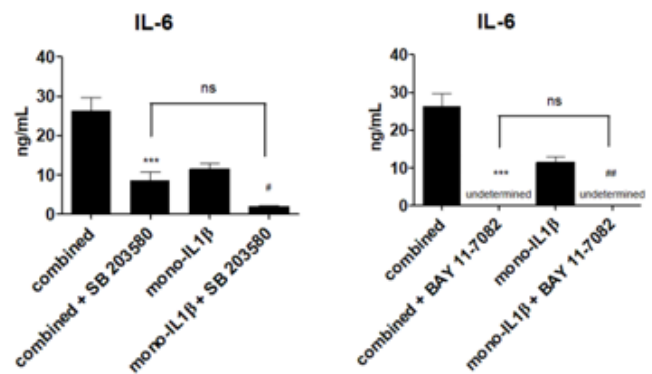

IL-8

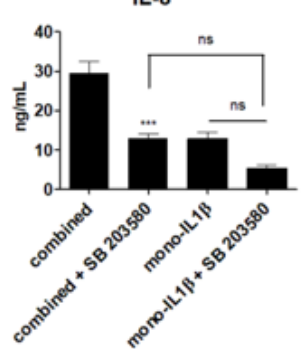

MMP-1

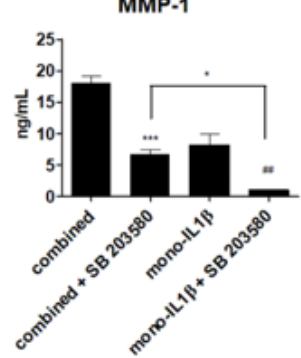

MMP-3

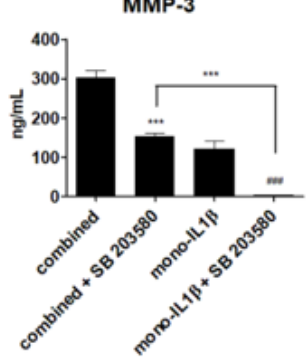

MMP-13

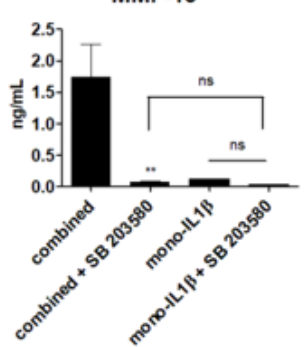

$L-8$

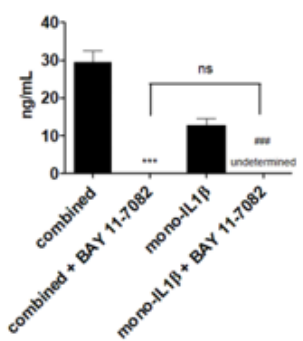

MMP-1

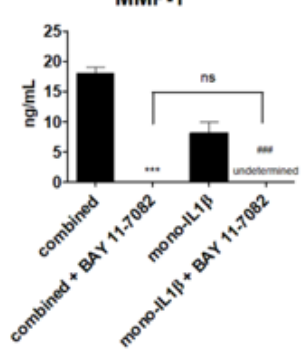

MMP-3

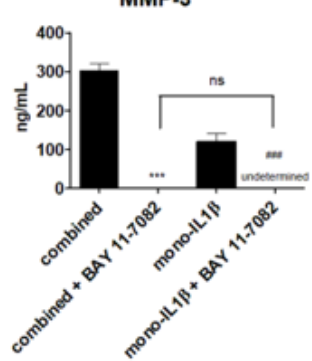

MMP-13

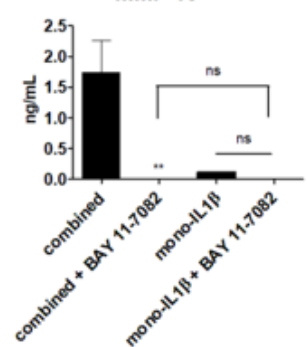

Figure 7

Combined-hResistin and IL-1 $\beta$ stimulation induced changes in the expression of secreted inflammatory cytokines and ECM catabolic enzymes ( ${ }^{*}<0.05$, $* \star p<0.01$, and $* \star \star ~ p<0.001$ compared to the combined stimulation group, $\# p<0.05$, \#\# $p<0.01$, and \#\#\# $p<0.001$, compared to the mono-IL1 $\beta$ stimulation group) (A) IL-6, (B) IL-8, (C) MMP-1, (D) MMP-3, and (E) MMP-13 in hAF cells, followed by 


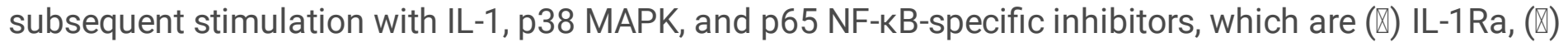
SB203580, and (『) BAY 11-7082.

( I ) hNP - IL-1Ra

(A)

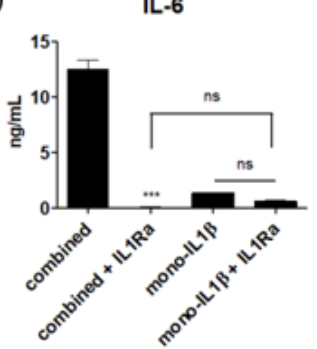

(B)

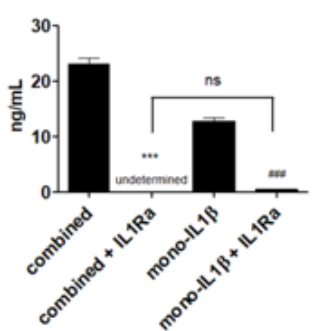

(C)

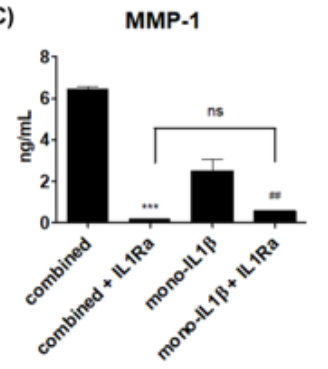

(D)

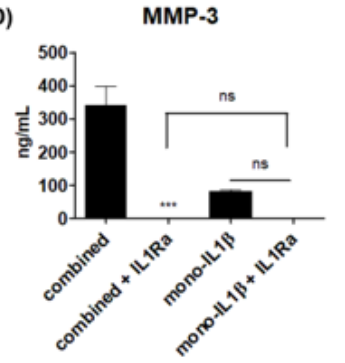

(E)

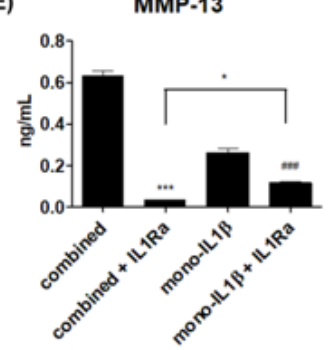

(II) hNP - SB203580

(III) hNP - BAY 11-7082

L-6

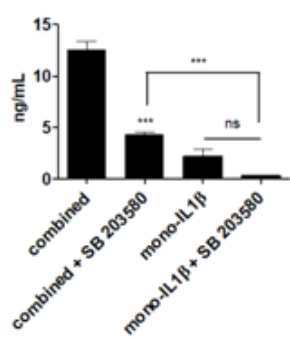

IL-8

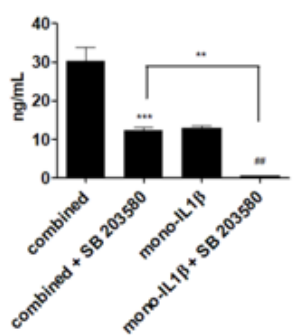

MMP-1

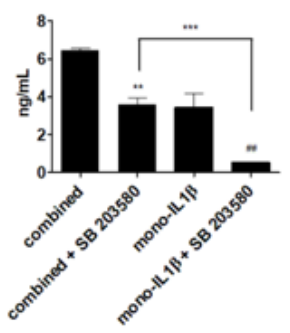

MMP-3

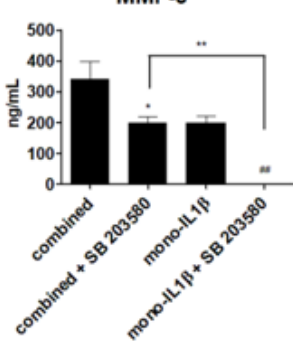

MMP-13

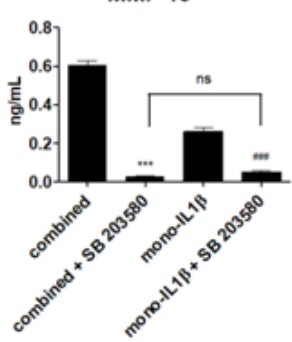

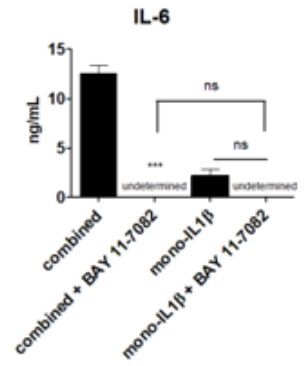

IL-8

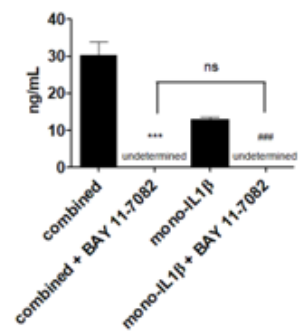

MMP-1

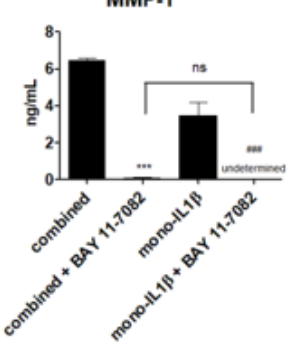

MMP-3

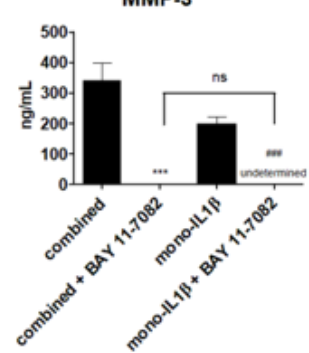

MMP-13

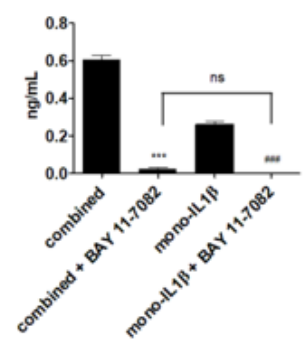

Figure 8

Combined-hResistin and IL-1 $\beta$ stimulation induced changes in the expression of inflammatory cytokines and ECM catabolic enzymes $\left(* p<0.05, * \star p<0.01\right.$, and ${ }^{\star * \star} p<0.001$ compared to the combined stimulation group, \#\# p $<0.01$ and \#\#\# p 0.001 compared to the mono-IL1 $\beta$ stimulation group) (A) IL-6, 
(B) IL-8, (C) MMP-1, (D) MMP-3, and (E) MMP-13 in hNP cells, followed by subsequent stimulation with IL1, p38 MAPK, and p65 NF-KB specific inhibitors, which are (『) IL-1Ra, (『) SB203580, and (囚) BAY 11-7082. 